\title{
INFRASTRUCTURE SHARING MODEL TO CONNECT THE UNCONNECTED IN RURAL AREAS
}

\author{
Ida Sèmévo TOGNISSE ${ }^{1}$, Ahmed Dooguy KORA ${ }^{2}$, Jules DEGILA ${ }^{1}$ \\ ${ }^{1}$ Institut de Mathématiques et Sciences Physiques, Porto-Novo, Benin, ${ }^{2}$ Ecole Supérieure Multinationale des \\ Télécommunications Dakar, Senegal
}

NOTE: Corresponding author: Ida Sèmévo TOGNISSE, ida.tognisse@imsp-uac.org

\begin{abstract}
One of the major problems the telecommunication industry faces in providing connectivity to the unconnected, particularly in rural and remote areas, is the lack of infrastructure in these areas. Indeed, deploying a network in an isolated area can be more expensive for an operator than in an urban area, while the return on investment is not possible. This is the primary cause of the coverage divide. To remedy this, in this work, we propose a techno-economic analysis of infrastructure sharing. First, we develop a mathematical model of the overall cost of extending a mobile network in rural areas. Different scenarios involving infrastructure sharing at varying levels of deployment are then presented. Then, using the models proposed in each scenario, we make a case study to deduce the most economically advantageous scenario for operators to extend their networks to remote areas. This case involves the sharing of passive infrastructure and also the sharing of active resources in a cloud-RAN. Based on the proposed model, our simulation results show that while passive sharing is beneficial, active sharing using cloud-RAN as technology increases this benefit. This work also indicates and highlights the technical constraints to be respected in the sharing for this scenario.
\end{abstract}

Keywords - Digital divide, infrastructure sharing, rural areas, total cost of ownership

\section{INTRODUCTION}

Around the world, the number of Internet users is increasing. New services, innovative applications, unique opportunities are offered through the Internet. But the digital divide still exists. The digital divide is defined as the separation between those who are connected and have access to services and those who are not. According to Global System for Mobile Communications Association (GSMA) reports, 46 percent of the world's population is still affected by the digital divide [1]. Typically, these populations are those living in poor, isolated and rural areas [2]. Indeed, one of the main causes of the digital divide is the lack of infrastructure in the regions. The direct consequence of the lack of infrastructure in the areas is mobile telephony, a high cost of deployment for the operators. For example, to extend a mobile network, a cell tower can, on average, costs between $\$ 150,000$ and $\$ 250,000$, base station equipment (baseband processors, transceivers, power supplies, amplifiers, etc.) between $\$ 20,000$ and $\$ 50,000$, installation of the equipment another $\$ 25,000$, and $\$ 5,000$ for interconnection to the core network [3]. So, it is a relatively expensive investment. However, if many operators can share the costly elements, this could result in substantial savings that can be used to extend the network in areas not covered [4]. This is why in the GSMA and International Telecommunication Union (ITU) recommendations for bridging the digital divide, the association suggests infrastructure sharing by operators $[5,6]$. Infrastructure sharing is a topic that is increasingly discussed in the literature, and its many benefits are of particular interest in the context of the extension and deployment of the latest generation of mobile networks $[7,8,9]$. In this paper, the purpose is to set up a mathematical model of infrastructure sharing by operators in the sense of universal access to technologies. Several scenarios are analysed to determine the most advantageous model for reducing the digital divide in rural areas. To do this, we started from existing sharing techniques and built scenarios using combinatorial methods. We first propose mathematical models to calculate the Total Cost of Ownership (TCO) to measure the benefits of infrastructure sharing, to carry out a comparative analysis of the scenarios to suggest the best alternative from a financial point of view. To do this, we started from the following research questions:

- What are the existing infrastructure sharing mechanisms?

- How do operators adopt these mechanisms?

- Which technologies can facilitate infrastructure sharing as a whole?

- What is the most suitable sharing scenario for poor and isolated rural areas to accelerate bridging of the digital divide?

To answer these questions, the rest of this paper is organised as follows. First, we present a literature review of different sharing techniques, technologies facilitating active sharing, cases of infrastructure sharing by operators, benefits obtained and mathematical models of sharing. In a second step, we model the TCO cost of investment according to several defined sharing scenarios. Then in the last part, we make a case study to examine these different sharing techniques to deduce the most appropriate one to connect poor and isolated rural areas. 


\section{LITERATURE REVIEW}

Infrastructure sharing in telecommunications refers to using the infrastructure or resource by more than one operator through an arrangement $[9,10]$. It is a process that offers many options. Sharing decisions range from sites, towers to another network infrastructure such as RAN and spectrum. According to the literature, there is no standard for infrastructure sharing, but it is considered a practical and promising solution for reducing deployment costs [4]. The main advantages of infrastructure sharing are the cost benefits $[11,12]$. But it is also driven by the migration to new technologies and the deployment of mobile broadband [13]. Infrastructure sharing models are divided into two broad categories: active and passive sharing.

\subsection{The different infrastructure sharing tech- niques}

Infrastructure sharing models are divided into two broad categories: active and passive sharing.

Passive sharing: This is a simple sharing model that does not present technical difficulties.

Site sharing: This is the sharing of the geographical location of stations. In this case, all network components at the site belong to the operators, respectively. This mode essentially saves the cost of leasing or purchasing and the cost of operating the territory. But the difficulty in this model is to find a fixed location that suits everyone to deploy the site when it is a new site.

Tower sharing: In this form of sharing, the site is shared by several operators, and the towers are also shared. Each operator deploys its own equipment and has control over it. The passive sharing agreement can be concluded between two or more operators and include third-party companies [14] which are neutral hosts. A neutral host refers to a telecommunication service provider interested in creating additional revenues and sharing sites or masts. In these cases, costs can be significantly reduced when several network operators or service providers share physical assets and transport networks.

Sharing can be managed by the site owner, who acts as a landowner for the operators who lease the site. The owner may be an operator sharing the site or another structure that provides the infrastructure.

Active sharing: Here, sharing extends to the electronic components of the network and the radio spectrum. A distinction is made between:

RAN sharing: The shared equipment includes BTS, NodeB, BSC, RNC... and may extend to feeder cables and antennas. But the transmission network and the core network are independent. Thus, this sharing mode allows operators to control their cells in their core network and have a separate operation [14].

Backhaul sharing: In addition to the RAN infrastructure, operators may decide to share the transmission channel. This is useful to extend their coverage more quickly and to focus on providing quality services to users. For backhaul sharing, several scenarios can be considered: The backhaul can be deployed by a joint venture of the participating mobile operators or a private entity would deploy and operate the infrastructure and offer it to the operators in a "platform as a service" model [15].

Core network sharing: Here, even the Home Location Register (HLR), the billing platform and the value-added system can be shared.

Active sharing in general and core network sharing is very complicated to achieve, especially when the infrastructure parties are competitors. Despite these benefits, infrastructure sharing can be a source of imbalance and market distortion and, if imposed from outside, can reduce incentives to build new infrastructure and adopt new technologies[10].

Several countries worldwide have approved passive infrastructure sharing between operators, but some network operators show resistance to opt for sharing so far. In [4], a survey on infrastructure sharing by operators was conducted. The results showed that infrastructure sharing among operators is deficient; mainly, site sharing is the most adopted. However, a techno-economic analysis of infrastructure sharing presented by [7] determined that it is not theoretically green and viable for a mobile network operator to own a tower alone in the case of deploying new technologies such as 5G; it could take more than 10 years to get a return on investment.

In rural areas, the cost of operating and maintaining rural sites grows exponentially with the remoteness of the sites and the lack of adequate infrastructure to access the sites [7]. But when network providers sell their towers, the cost-benefit ratio becomes more attractive $[7,10]$. In Madagascar, a country where two-thirds of the population live in rural areas, the adoption of passive sharing and backhaul by all mobile operators according to open access principles has enabled rapid, efficient and cost-effective mobile network expansion in rural areas [10]. Despite the technical and political difficulties of implementing active sharing, while passive sharing leads to significant savings, active sharing to some extent can significantly increase these savings especially when appropriate technology is used for sharing.

\subsection{Cloud-RAN and network slicing as an ac- tive sharing option for bridging the digital divide}

The concept of cloud-RAN refers to a radio access network in which BaseBand Units (BBUs) are centralized at a geographical location such as a data center using cloud computing and virtualization techniques. Remote Radio Head (RRH) is deployed with antennas at the sites and is connected to the BBU pool via a fronthaul link. The BBU pool provides all baseband signal processing (transmission, centralized resource allocation, joint user scheduling, flow control data, etc.) in the cloud-RAN. In contrast, conventional base stations, which are replaced by 
low-cost RRH, only provide low-complexity transmission and reception. Due to its low complexity functionality, its size is smaller than that of conventional base stations and can be easily installed [16]. The BBU pool is deployed on hardware, i.e. multi-core servers, and the baseband functions are software-defined and run as applications on the servers [17].

The capacity of the cloud is defined by the number of BBUs hosted on the servers. The BBU pools can be stacked without a direct link or interconnected in such a way as to share resources and functionality according to demand from the RRH. Therefore, if $\mathrm{n}$ is the number of BBUs hosted in the cloud, it is possible to perform baseband processing of $m$ RRH. In the case of rural areas, it can be seen that when a network is traditionally deployed, the traffic is low; the network is under exploited because of the low population density. As a result, $\mathrm{m}$ can become very large compared to $n$. The cloud-RAN also allows the coexistence of several technologies. This will be very important to offer voice telephony services with $2 \mathrm{G}$ infrastructure, for example, and broadband with $4 \mathrm{G}$ to several cells simultaneously. Thus, pooling the resources deployed and sharing these resources in the cloud can benefit the operators and enable the efficient and optimal use of the deployed resources. In [18], it was shown that the more cells or base stations that use a cloud-RAN, the lower the overall CAPEX deployment cost and TCO per site. And the faster the return on investment in the particular case of rural areas. Thus, by opting for joint construction of a cloud-RAN in which several operators share the infrastructure, it accelerated the reduction of the digital bill.

On the other hand, a new paradigm brought about by $5 \mathrm{G}$ now makes it possible to consider sharing in the network according to several services: network slicing. The network slicing consists of using virtualization, i.e. Software Defined Network (SDN) and Network Function Virtualization (NFV), to partition and optimize resource management by creating slices. The network slice is a logical network that can be technically deployed in all fixed and mobile networks [19].

Network slicing allows for different performances associated with each slice and allocates dedicated resources per type of usage. Each network slice, therefore, corresponds to a specific use different from the other slices. The network slice can be considered as a real catalyst for services. It can be created, modified and deleted using network management functions [20]. In particular, network slicing as a form of resource sharing in the RAN is a mechanism that allows the sharing of a single network infrastructure between several operators, where each operator provides its functionality and services.

Different sharing techniques can lead to significant savings. However, the most important thing is to find an optimal model for sharing, especially in a context where the return on investment is not obvious, such as in rural, poor and remote areas. In the literature, different approaches and models have been proposed to assess the contribution of sharing techniques. [14], different scenarios of infrastructure sharing models are explored to build a mathematical model to analyze the benefits and costs of infrastructure sharing in China. Vanessa Vasconcellos et al. also proposed a mathematical model to calculate the benefits of infrastructure sharing between operators and neutral hosts in $5 \mathrm{G}$ based on cost evaluation [8]. This study aims to set up a framework based on the comparison with other sharing strategies, suggesting the best alternative from a cost point of view. Using urban environments with very high population density as a case study in which several actors share passive infrastructure, preliminary results have shown that sharing with a neutral host can increase savings compared to other passive sharing strategies. Based on game theory, Adrian Kliks [21] found that operators can achieve substantial financial savings by avoiding new site construction costs at overlapping sites, consolidating existing sites, and reducing lease, maintenance and transmission costs.

In Joseph [3] an extensive analysis of infrastructure sharing and a model to be developed to analyze the cost and benefits of tower sharing in Ghana. The results obtained from data sources support the propositions of the literature review that infrastructure sharing provides operators with a wide range of benefits and savings. On average, about $44.61 \%$ of capital can be saved if the eight telecom operators in the country share a tower. In [9], the authors propose a model for infrastructure sharing and found that sharing can save between 30 and 40 percent of CAPEX and OPEX expenses. Also, [22] has developed a model for backhaul sharing using different technologies. However, to better appreciate the benefits of sharing, a complete model that considers all the modules for network extension is required. Also, in the various studies, the modelling is done for passive sharing in the RAN. The study cases are generally for densely populated areas where the network capacity implemented on the sites is fully utilized. Although we have a significant amount of modelling, the authors do not consider infrastructure sharing an application framework for universal ICT access or removing the digital bill. Therefore, in this study, we model passive and active infrastructure sharing and network resource sharing to eliminate the rural digital divide.

\section{INFRASTRUCTURE SHARING MODEL}

This section presents a modelling approach for infrastructure sharing solutions in mobile network extension in rural areas. This modelling calculates the total investment cost resulting from infrastructure deployment according to the sharing approach. For this purpose, we use the OPEX and CAPEX cuts to determine the TCO.

The first step of our framework consists of a total network deployment cost model in the standard case as presented in Fig.1. 


\section{Scenario 1: Standard case}

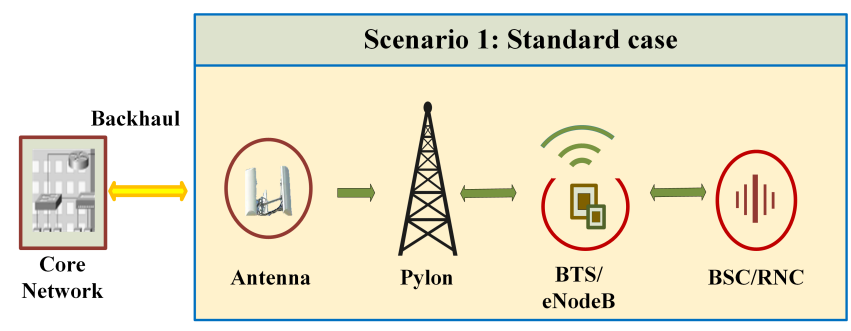

Fig. 1 - Standard case of network extension

In this case, for network extension, the total cost of ownership for each operator is given by:

$$
T C O=T C O_{R A N}+T C O_{\text {backhaul }}
$$

TCO : Total Cost of Ownership for network $T C O_{R A N}$ : Total Cost of Ownership for RAN $T C O_{\text {backhaul }}$ : Total Cost of Ownership for backhaul

$$
T C O_{R A N}=C_{C a p R A N}+C_{o p R A N}+C_{\text {spectre }}
$$

$C_{\text {Cap RAN }}$ : CAPEX cost of RAN

$C_{\text {op RAN }}$ : OPEX cost of RAN

$C_{\text {spectre }}$ : cost of the diffusion spectrum in the RAN

Firstly, the cost of the spectrum $C_{\text {spectre }}$ is neglected because it is assumed that the operator wishing to extend its network has at least a national licence to broadcast in the RAN.

Secondly, for the CAPEX, we consider the cost of the site, the cost of the tower, the cost of the base station itself and the cost of the electrical infrastructure.

$$
C_{\text {Cap RAN }}=C_{\text {site }}+C_{\text {pylon }}+C_{\text {eNodeB }}+C_{\text {energy }}
$$

With

$C_{\text {site }}$ : CAPEX of a site

$C_{\text {pylon }}:$ CAPEX of a pylon

$C_{e N o d e B}:$ CAPEX of a base station

$C_{\text {energy }}$ : CAPEX of energy

In fact, in one year, the OPEX cost related to the infrastructure (except energy equipment) can be estimated at a percentage $\alpha$ and, depending on the year, there is a reduction of the OPEX investment by a coefficient $r$ due to the knowledge and mastery of the deployed system. Also, the energy OPEX is equal to a percentage $\beta$ of the energy CAPEX [23]. Thus, integrating these elements, we obtain: For the first year:

$$
C_{\text {op } 1}=\alpha \cdot\left(C_{\text {site }}+C_{\text {pylon }}+C_{\text {eNodeB }}\right)+\beta \cdot C_{\text {energy }}
$$

Let be: $C o_{1}=\alpha \cdot\left(C_{\text {site }}+C_{\text {pylon }}+C_{\text {eNodeB }}\right)$

After this year,

$$
\begin{aligned}
C o_{2} & =(1-r) \cdot C_{o 1} \\
C_{0} o_{3} & =(1-r) \cdot C_{o 2}=(1-r)^{2} \cdot C_{o 1} \\
C o_{k} & =(1-r) \cdot C_{o k-1}=(1-r)^{k-1} \cdot C_{o 1}
\end{aligned}
$$

The OPEX cost in T year given:

$$
C_{\text {op } T}=\alpha \sum_{k=1}^{T}(1-r)^{k-1}\left(C_{\text {site }}+C_{\text {pylon }}+C_{\text {eNodeB }}\right)+\beta . T . C_{\text {energy }}
$$

The TCO of the RAN gives:

$$
\begin{gathered}
T C O_{R A N}=\left(C_{\text {site }}+C_{\text {pylon }}+C_{\text {eNodeB }}\right)\left(1+\alpha \sum_{k=1}^{T}(1-r)^{k-1}\right)+ \\
(1+\beta . T) C_{\text {energy }}
\end{gathered}
$$

For the backhaul, we have:

$$
T C O_{\text {backhaul }}=C_{\text {caplink }}+C_{\text {oplinkT }}+C_{\text {spectre }}
$$

$C_{\text {spectre }}$ : Annual cost of spectrum

$C_{\text {caplink }}$ : CAPEX cost of transmitter-receiver equipment $C_{\text {oplinkT }}$ : OPEX cost of transmitter-receiver equipment. By considering that the OPEX cost of the link is equal to a percentage $\omega$ of the CAPEX cost and that according to the time one notes a reduction of the cost of a coefficient $r^{\prime}$ one obtains the TCO backhaul cost which is given by:

$$
\begin{gathered}
\left.T C O_{\text {backhaul }}=C_{\text {caplink }}+T . C_{\text {spectre }}+\omega \sum_{k=1}^{T}\left(1-r^{\prime}\right)^{k-1}\right) C_{\text {caplink }} \\
T C O_{\text {backhaul }}=\left(1+\omega \sum_{k=1}^{T}\left(1-r^{\prime}\right)^{k-1}\right) C_{\text {caplink }}+T . C_{\text {spectre }}
\end{gathered}
$$

$$
\begin{gathered}
\text { TCO }=\left(C_{\text {site }}+C_{\text {pylon }}+C_{\text {eNodeB }}\right)\left(1+\alpha \sum_{k=1}^{T}(1-r)^{k-1}\right)+ \\
(1+\beta . T) C_{\text {energy }}+\left(1+\omega \sum_{k=1}^{T}\left(1-r^{\prime}\right)^{k-1}\right) C_{\text {caplink }}+T . C_{\text {spectre }}
\end{gathered}
$$

For $n$ site, we have:

$$
\begin{gathered}
T C O(n)=n\left(C_{\text {site }}+C_{\text {pylon }}+C_{\text {eNodeB }}\right)\left(1+\alpha \sum_{k=1}^{T}(1-r)^{k-1}\right)+ \\
\left(1+\omega \sum_{k=1}^{T}\left(1-r^{\prime}\right)^{k-1}\right) n . C_{\text {caplink }}+(1+\beta . T) n . C_{\text {energy }}+T \cdot C_{\text {spectre }}
\end{gathered}
$$

The next step is to calculate the cost according to different sharing scenarios of interest in the context of rural areas. In these scenarios, we consider sharing the infrastructure by $\mathrm{m}$ operators and deploying $\mathrm{n}$ shared sites.

\section{Scenario 2: Site sharing}

In this case, the TCO formula becomes:

$$
\begin{gathered}
T C O(n)=n\left(\left(\frac{1}{m}\right) C_{\text {site }}+C_{\text {pylon }}+C_{\text {eNodeB }}\right)\left(1+\alpha \sum_{k=1}^{T}(1-r)^{k-1}\right)+ \\
\left(1+\omega \sum_{k=1}^{T}\left(1-r^{\prime}\right)^{k-1}\right) n . C_{\text {caplink }}+(1+\beta . T) n . C_{\text {energy }}+T . C_{\text {spectre }}
\end{gathered}
$$




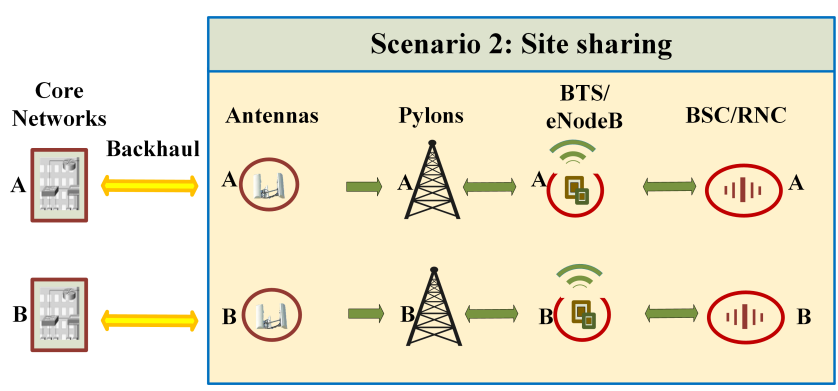

Fig. 2 - Site sharing by operators

\section{Scenario 3: Pylon sharing}

Let us now consider, in addition to site sharing, tower sharing at each site.

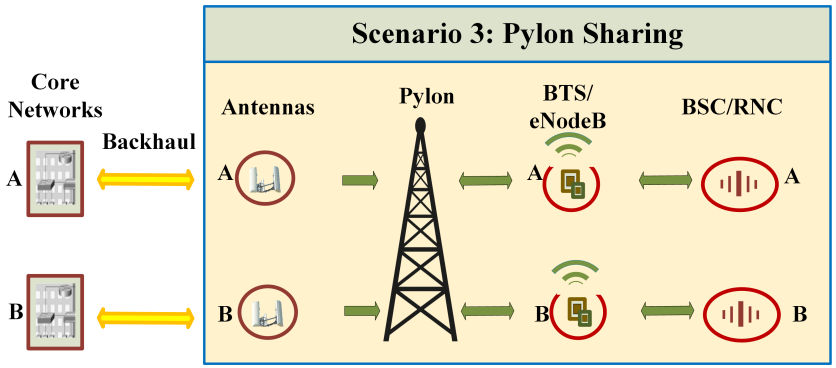

Fig. 3 - Sites and pylon sharing by operators

$$
\begin{gathered}
T C O(n)=n\left(\left(\frac{1}{m}\right) C_{\text {site }}+\left(\frac{1}{m}\right) C_{\text {pylon }}+C_{\text {eNodeB }}\right)\left(1+\alpha \sum_{k=1}^{T}(1-r)^{k-1}\right)+ \\
\left(1+\omega \sum_{k=1}^{T}\left(1-r^{\prime}\right)^{k-1}\right) n . C_{\text {cap }, \text { link }}+(1+\beta . T) n . C_{\text {energy }}+T . C_{\text {spectre }}
\end{gathered}
$$

\section{Scenario 4: Backhaul sharing}

Let us now consider a scenario where operators join forces to build a common backhaul such as fibre or HighAltitude Platform Systems (HAPS) to go together to unserved areas. The operators share the CAPEX and OPEX costs. So the bandwidth of the platform is divided between the operators for their personal use. The transmission infrastructure to be deployed will carry the various signals to and from the sites using appropriate multiplexing techniques.

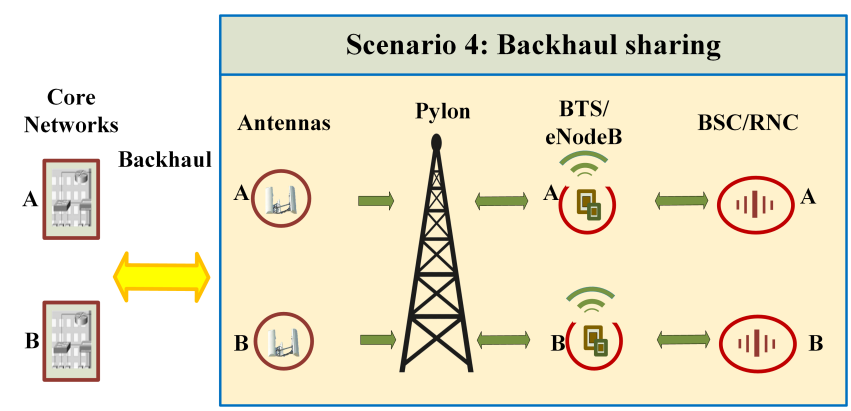

Fig. 4 - Backhaul sharing

$$
\begin{aligned}
& T C O(n)=n\left(\left(\frac{1}{m}\right) C_{\text {site }}+\left(\frac{1}{m}\right) C_{\text {pylon }}+C_{\text {eNodeB }}\right)\left(1+\alpha \sum_{k=1}^{T}(1-r)^{k-1}\right)+ \\
& \left(1+\omega \sum_{k=1}^{T}\left(1-r^{\prime}\right)^{k-1}\right) n \cdot\left(\frac{1}{m}\right) \cdot C_{\text {caplink }}+(1+\beta \cdot T) n \cdot C_{\text {energy }}+T \cdot C_{\text {spectre }}
\end{aligned}
$$

\section{Scenario 5: Cloud-RAN}

Finally, let's consider the scenario where operators join forces to build a cloud and a dedicated front-end link in which sites and masts are shared.

In [18], it is shown that, in a standard case, C-RAN is not more beneficial than traditional RAN in the context of rural areas. However, with the underutilization of resources and the possibility that C-RAN offers to share the resource in the BBU pool, C-RAN becomes beneficial when doing a massive deployment. This is what justifies the choice to use a shared C-RAN

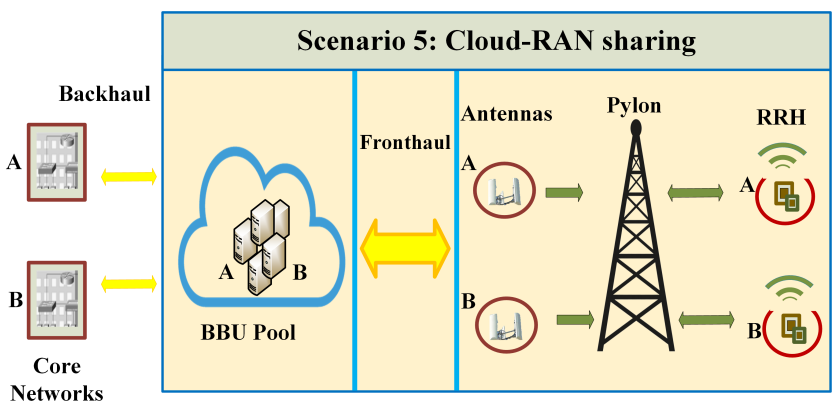

Fig. 5 - Cloud-RAN sharing

In this scenario, another entity is added to the network; it is the fronthaul with a cost model similar to that of the backhaul. Considering that the cost of an eNodeB is the sum of the cost of a BBU and that, as shown in [24], thanks to the cloud, we have a gain in global energy expenditure, we have :

$$
\begin{gathered}
T C O(n)=\left(\left(\frac{n}{m}\right) C_{\text {site }}+\left(\frac{n}{m}\right) C_{\text {pylon }}+n^{\prime} \cdot C_{B B U}+n \cdot C_{R R H}\right) \\
\left(1+\alpha \sum_{k=1}^{T}(1-r)^{k-1}\right)+\left(1+\omega^{\prime} \sum_{k=1}^{T}\left(1-r^{\prime \prime}\right)^{k-1}\right) \\
n \cdot\left(\frac{1}{m}\right) \cdot C_{\text {cap fronthaul }}+(1+\beta \cdot T) n \cdot \Delta \cdot C_{\text {energy }}+T \cdot C_{\text {spectre }}+C_{\text {sup }}
\end{gathered}
$$

$C_{\text {cap fronthaul }}$ : CAPEX cost of transmitter-receiver equipment on fronthaul link

$C_{\text {sup }}$ : the additional cost generated by the cloud.

$\Delta:$ the coefficient that is induced by the energy gain $n$ ': is number of BBUs

In rural areas, the traffic used in the cells is generally very low. Centralization of the BBU can allow efficient use of the resource in that, one BBU can support the traffic of several RRH through virtualization. Also, half of a BTS site can be used for one RRH site. Let $x$ be the number of RRHs using the resource equivalent of one physical BBU. 


$$
\begin{gathered}
T C O(n)=\left(\left(\frac{n}{m}\right) C_{\text {site }}+\left(\frac{n}{m}\right) C_{\text {pylon }}+\left(\frac{n}{x}\right) \cdot C_{B B U}+n \cdot C_{R R H}\right) \\
\left(1+\alpha \sum_{k=1}^{T}(1-r)^{k-1}\right)+\left(1+\omega^{\prime} \sum_{k=1}^{T}\left(1-r^{\prime \prime}\right)^{k-1}\right) \\
n \cdot\left(\frac{1}{m}\right) \cdot C_{\text {cap fronthaul }}+(1+\beta \cdot T) n \cdot \Delta \cdot C_{\text {energy }}+T \cdot C_{\text {spectre }}+C_{\text {sup }}
\end{gathered}
$$

Although this scenario allows for asset sharing, it should be noted that the centralisation of BBU presents certain challenges, one of the main ones being the fronthaul requirements. Indeed, this link's time budget or allowable latency is $1 \mathrm{~ms}$ and $2 \mathrm{~ms}$ for the downlink and uplink, respectively [17]. Because of this constraint, many studies propose fibre deployment for the fronthaul $[25,26]$. However, in the context of remote areas, this is not appropriate for several reasons. These areas are sometimes challenging to access, and fibre deployment is costly for these areas.

The limitation that can be observed in the latter scenario is that active resource sharing induces additional latency in the network. The latency is the time for a packet to leave point A to point B. Several elements cause this time: we distinguish the latency related to the processing at each link node and the latency associated with the propagation. This is because sharing processing resources results in additional latency, a source of inefficiency or performance degradation [27]. Also, the latency/jitter introduced by the fronthaul link is also a significant

\begin{tabular}{|c|c|c|c|c|}
\hline Technologies & Bandwidth & Modulation & $\begin{array}{l}\text { Theoretical } \\
\text { through- } \\
\text { put }\end{array}$ & $\begin{array}{l}\text { Latency } \\
\text { in } \mathrm{ms}\end{array}$ \\
\hline $\begin{array}{ll}2.75 & \mathrm{G} \\
\text { :EDGE } & \\
\end{array}$ & $\overline{200 \mathrm{Khz}}$ & $\begin{array}{l}16 \text { et } \\
32 \mathrm{QAM}\end{array}$ & $384 \mathrm{~kb} / \mathrm{s}$ & 150 \\
\hline $3 \mathrm{G}:$ UMTS & $5 \mathrm{Mhz}$ & 16QAM & $1.9 \mathrm{Mb} / \mathrm{s}$ & 100 \\
\hline $\begin{array}{l}3.5 \mathrm{G}: \mathrm{HS}- \\
\text { DPA }\end{array}$ & $5 \mathrm{Mhz}$ & 64QAM & $14.4 \mathrm{Mb} / \mathrm{s}$ & 50 \\
\hline $3.9 \mathrm{G}:$ LTE & $\begin{array}{l}1.4 \mathrm{Mhz}- \\
20 \mathrm{Mhz}\end{array}$ & $\begin{array}{l}256 \text { QAM/ } \\
\text { OFDM }\end{array}$ & $326 \mathrm{Mb} / \mathrm{s}$ & 10 \\
\hline 4 G : LTE-A & $\begin{array}{l}1.4 \text { Mhz- } \\
20 \mathrm{Mhz}\end{array}$ & $\begin{array}{l}256 \text { QAM } \\
\text { OFDM } \\
\text { MIMO }\end{array}$ & $1 \mathrm{~Gb} / \mathrm{s}$ & 5 \\
\hline $5 \mathrm{G}$ & $\begin{array}{l}\text { 1.25 Mhz- } \\
20 \mathrm{Mhz}\end{array}$ & $\begin{array}{l}256 \text { QAM } \\
\text { No OFDM }\end{array}$ & $10 \mathrm{~Gb} / \mathrm{s}$ & 1 \\
\hline
\end{tabular}
limitation for future C-RAN-based wireless systems [28].

Table 1 - Characteristics of mobile network technologies

When leaving a mobile network evolution for another one, several changes are made, notably in the control plane, to reduce processing latency. Also, evolving a modulation order and increasing the bandwidth allows for increasing the throughput in the network. In general, depending on the mobile network generation, there is an allowable latency that should not be exceeded and a theoretical bit throughput associated with it. For example, in EDGE, the latency is 150 milliseconds (ms) for a theoretical bit throughput of 384 kilobits per second $(\mathrm{kb} / \mathrm{s})$, and in LTE, the latency is $10 \mathrm{~ms}$ for a theoretical bit throughput of 326 megabits per second (Mb/s). Table 1 presents the characteristics of different mobile network evolution.

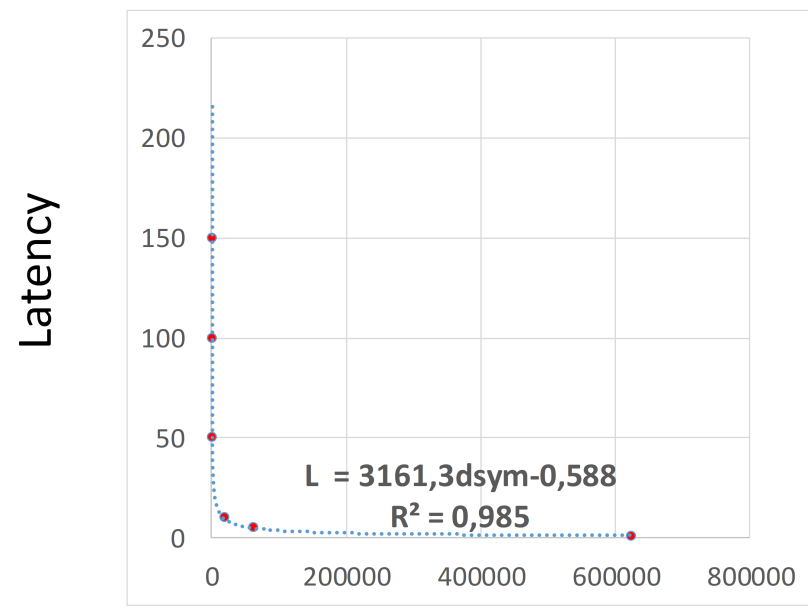

Symbol Throughput

Fig. 6 - Variation of latency as a function of symbol throughput according to different mobile network evolution.

When we look at how the latency evolves from one evolution to another depending on the symbol throughput in the network, we get the points in Fig.6.

These different points can be used to approximate theoretically the latency and the symbol rate by a power function defined by the function in Fig. 6.

These different points can be used to theoretically approximate the latency and the symbol throughput by a function defined by: $L=3161,3 \mathrm{dsym}^{-0.588}$ with a statistical measure of the closeness of the data to the fitted regression line evaluated at $R^{2}=92.14 \%$.

$\mathrm{L}$ is the latency in the network in millisecond (ms), $d_{\text {sym }}$ is the symbol throughput in megasymbol per millisecond (Msym/ms).

The symbol throughput being obtained by dividing the bit throughput by the modulation order.

This formula can be considered an approximation of how latency evolves in a mobile network due to symbol throughput variation. This means that theoretically when we vary the data rate in the operator's network, we have a parallel influence on the latency. However, in a cloud-RAN, the direct consequence of sharing the active resource as a BBU is sharing the operator's dedicated throughput. This throughput is limited in the case of resource load sharing. This implies an additional latency on top of the latency in the network. On the other hand, the centralized BBU adds to the front-end network, which can also influence the latency. Indeed, suppose the signal propagation speed $V=D / T p$, depending on the operating distance between the cloud and the BBU or the transmission medium used. In that case, there will also be an additional time added in terms of latency in the network. $\mathrm{V}$ is the propagation speed of the signal, D is the path 
distance of the signal on the fronthaul link depending on the type of technology used and the network topology. If in the standard case, the throughput is dsym, and the cloud, the processing unit in the cloud takes care of processing of $\mathrm{xRRH}$, the additional latency in the network is given by:

$$
\begin{aligned}
& L_{a d d}=3161,3\left(\frac{d s y m}{x}\right)^{-0,588}-3161,3 d \text { sym }^{-0,588}+\frac{D}{V} \\
& L_{a d d}=3161,3 d \text { sym }^{-0,588}\left(x^{0,588}-1\right)+\frac{D}{V}
\end{aligned}
$$

$L_{a d d}:$ additional latency that this scenario induces in the network.

$\mathrm{D}$ in metre $(\mathrm{m})$ and $\mathrm{V}$ in metre per millisecond $(\mathrm{m} / \mathrm{ms})$. In sum, in scenario 5 , this latency constraint must be taken into account by minimizing $L_{a d d}$.

\section{CASE STUDY AND RESULT}

The third and final step is to determine the most appropriate sharing case for rural areas. For this, we will proceed by applying the different models proposed above to a case study of deployment in rural areas.

These costs are chosen by considering the approximate cost of the equipment at the time of purchase. These are indicative values that may vary from one manufacturer to another. Also the parameters used can change from one region to another. It is considered in $[29,6]$ that, in a transmission system or a radio access network, the OPEX cost represents $5 \%$ of the CAPEX cost and on the other hand, that the OPEX cost in energy represents $25 \%$ of the CAPEX in energy. [30] has shown that we can have a benefit of more than $2 / 3$ of the energy consumption by switching from traditional RAN to cloud-RAN. As a result, for our application case, we use the parameters presented in Table 2.

Table 2 - Characteristics of mobile network technologies

\begin{tabular}{|c|c|}
\hline Parameters & Values \\
\hline $\mathrm{n}$ & 50 \\
\hline $\mathrm{m}$ & 3 \\
\hline $\mathrm{x}$ & 4 \\
\hline$C_{\text {eNodeB }}$ & $\$ 3000$ \\
\hline$C_{B B U}$ & $\$ 2000$ \\
\hline$C_{R R H}$ & $\$ 1000$ \\
\hline$C_{\text {site }}$ & $\$ 40000$ \\
\hline$C_{\text {pylon }}$ & $\$ 150000$ \\
\hline$C_{\text {energy }}$ & $\$ 30000$ \\
\hline$C_{\text {cap }}$ & $\$ 400000$ \\
\hline$C_{\text {cap fronthaul }}$ & $\$ 400000$ \\
\hline$C_{\text {sup }}$ & $\$ 100000$ \\
\hline$C_{\text {spectre }}$ & $\$ 200000$ \\
\hline$\alpha=\omega=\omega^{\prime}$ & 0.05 \\
\hline$\beta$ & 0.25 \\
\hline$\Delta$ & 0.66 \\
\hline $\mathrm{r}=\mathrm{r}^{\prime}=\mathrm{r}^{\prime \prime}$ & 0.05 \\
\hline
\end{tabular}

Fig. 7 shows the evolution of the TCO over 50 sites as a function of time for each scenario.

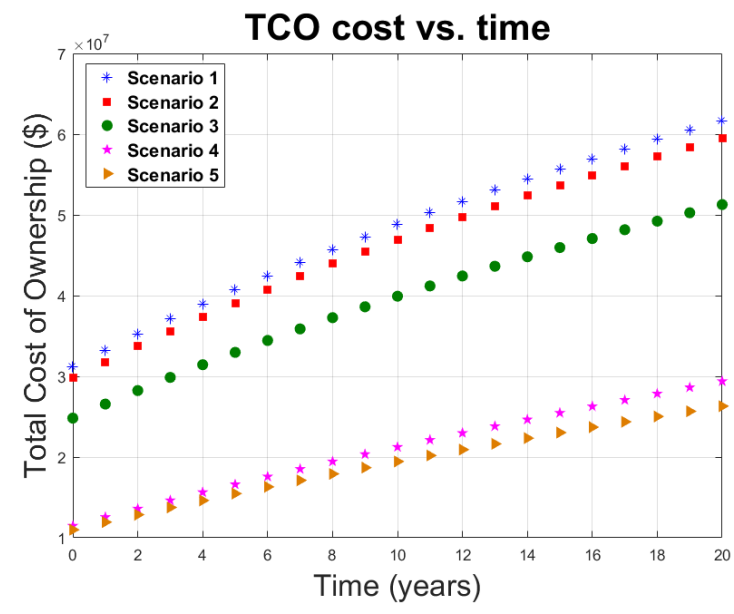

Fig. 7 - TCO over 50 sites

From Fig. 7 we can see that, whatever the sharing scenario, there is a reduction in TCO costs compared to the standard case defined by scenario 1 . The sharing of pillars and sites (scenario3) between the three operators significantly impacts the TCO. In line with the results of Ovase Karim et al. [9]. This case study shows that site and tower sharing between several operators generate CAPEX cost benefits of more than 40 percent. However, when we look at the scenarios integrating active sharing (scenarios 4 and 5), we see that the impact is even more significant and the benefits higher. The curve analysis allows us to say that the benefits are very significant, especially with scenario 5, where the site, the pylon, and a cloud-RAN are shared. Therefore, this scenario presents itself in our simulation as the best advantage from an economic point of view.

However, as mentioned above, sharing active resources through this scenario can have consequences on the network's performance, especially on latency. To see to what extent this scenario can be considered technically applicable, we will test the impact of sharing in this scenario. Fig. 8 shows the evolution of the additional latency in a cell when it shares the resource or its traffic through several cells and as a function of the distance separating it from the radio site of the $\mathrm{BBU}$ in the cloud. In this simulation, we consider that the radio frequency signal is transmitted at a frequency that allows it to propagate at a speed of $3.10^{8} \mathrm{~m} / \mathrm{s}$. Microwave, satellite or HAPS can therefore be considered. This is what makes that after simulation; we discuss the values of latency according to the technologies and those acceptable for the system. We notice that as the number of RRHs sharing the active resource increases, the additional latency in each cell increases similarly. We see that, in reality, it is the sharing of the resource that increases the latency. For a $\mathrm{D}=0 \mathrm{~km}$, depending on whether the number of RRHs varies from 1 to 6 , the latency runs from 0 to $9 \mathrm{~ms}$. Also, as the frontier distance increases, the additional latency increases to $10 \mathrm{~ms}$ over $300 \mathrm{~km}$ if $\mathrm{x}=6$. In our specific application case of scenario $5, x=4$, which means that the resource equivalent of one BBU is shared between about four RRHs. 


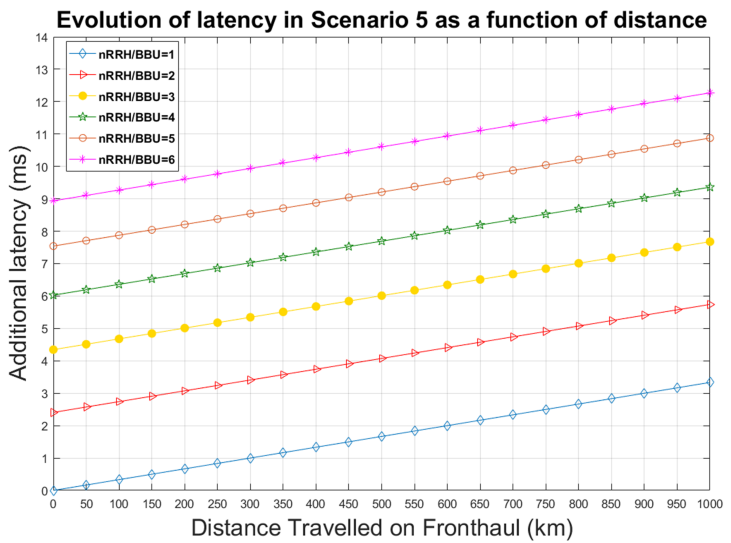

Fig. 8 - Latency evolution as a function of the number of RRHs supported by $\mathrm{BBU}$ resource and fronthaul distance

When the distance evolves the $100 \mathrm{~km}$ with each BBU shared between several RRHs, the latency time added in the network is from $6 \mathrm{~ms}$ to $6.4 \mathrm{~ms}$. Indeed, in the specific case of the rural and isolated areas, the techniques in fronthaul usable are the microwave, the high-altitude platform system, and the satellite because of the difficulty of access. For the microwave, knowing that it is a terrestrial link, we can say that the latency introduced will be about $6.4 \mathrm{~ms}$ if we stay within $100 \mathrm{Km}$ of the fronthaul. For the high-altitude platform system, given that they are generally located between 18 and $20 \mathrm{Km}$ from the ground [15], the latency introduced into the network will then be of the order of $6,2 \mathrm{~ms}$ for the round trip time. In summary, active infrastructure sharing, even if difficult to achieve, is possible with the cloud-RAN. This option is, from an economic point of view, the best in the different scenarios presented. Fig. 7 shows that as the level of sharing increases, the cost of deployment decreases, and Fig. 8 shows that sharing can lead to significant additional latency in the network, which can have consequences on performance. Indeed, to reduce the deployment cost significantly, an operator can share more of its active infrastructure. When we are interested in quantifying the relationship between the network deployment cost and the additional latency in the specific case of the selected scenario 5, we obtain the curve in Fig. 9. In our fronthaul simulation, we have considered a transmission by HAPS, which imposes a round trip of about $40 \mathrm{~km}$. We vary the number of operators sharing the infrastructure to determine the deployment cost per site and calculate the corresponding additional latency value in each case. This figure shows that the deployment cost can be considerably reduced thanks to sharing; however, the network performance can decrease significantly by prioritizing the cost reduction. It will then be necessary to find a good compromise between cost and performance. The additional latency introduced by the centralisation of BBU is significant, especially for technologies such as 4G and 5G. However, it will give a considerable boost to bridging the digital divide. Therefore, it must be allowed by primary legislation, encouraged by regulators voluntarily and adopted by operators to provide services.

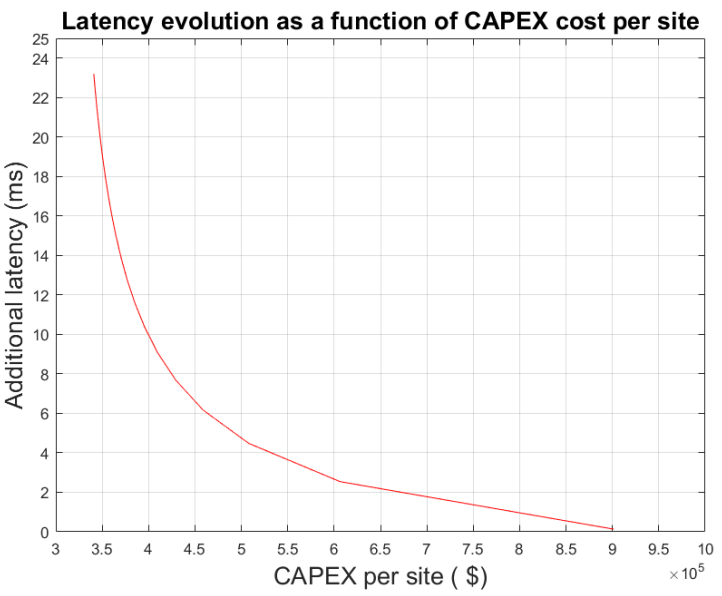

Fig. 9 - Evolution of latency as a function of deployment cost per site

\section{CONCLUSION}

In this paper, we focus on the evaluation of different infrastructure sharing techniques in mobile networks. For this purpose, we model the total investment cost for mobile network extension in different scenarios. The objective is to find the most advantageous scenario for connecting the unconnected and eliminating the digital divide. The main contributions of this paper are the formulation of a mathematical cost model for each infrastructure sharing technique, the proposal of an optimal sharing model, and the application of these models in the context and realities of rural, poor and isolated areas. The presented scenarios combine several infrastructure sharing techniques and propose a large-scale deployment involving several operators for a fast, efficient and cost-effective extension of ICT in general and mobile telephony and broadband, particularly in remote areas. After demonstrating the benefits of these scenarios, we show the constraints that must be respected to limit the impact on network performance using latency as a parameter. The main limitation of this work is the consideration of network performance indicators such as KPI and the feasibility and sizing of the network in a real environment. The other limitation of this paper is that it does not take into account spectrum sharing. The latter being a scarce and expensive resource, our future work will focus on this point more precisely by considering that white space is important in these regions. As a follow-up to this study, we are also undertaking field studies to evaluate the implementation of the chosen scenario to suppress the digital gap in two developing countries.

\section{ACKNOWLEDGEMENT}

We are grateful to the African Center of Excellence in Mathematical Science, Informatics, and Applications, IMSP, Dangbo, Republic of Benin, which supported this work. 


\section{REFERENCES}

[1] GSMA. "Driving the digital revolution with improved mobile coverage". In: September (2020).

[2] ITU. Telecommunications / ICTs for rural and remote areas. Tech. rep. 2017. URL: www . itu . int $\%$ $20 \%$ E $2 \% 80 \%$ BA $\% 20$ opb $\% 20 \%$ E $2 \% 80 \%$ BA $\% 20$ stg $\% 20 \%$ E2\%80\%BA\%20D-STG-SG01.05-2017-PDF-E.pdf.

[3] Joseph Kweku. "Analysis of Co-Location of Telecommunication Infrastructure in Ghana". In: Proc. - 2019 Int. Conf. Comput. Comput. Model. Appl. ICCMA 2019 (2019), pp. 72-80. DOI: 10.1109/ICCMA.2019.00019.

[4] S M M Gilani, A Kabir, I Ullah, and G Rehman. "Techno-Economic Analysis of Green and Sustainable Infrastructure Sharing in Mobile Communication Systems". In: 26.1 (2021), pp. 80-89.

[5] GSMA. Enabling Rural Coverage Regulatory and policy recommendations to foster mobile broadband coverage in developing countries. Tech. rep. 2018.

[6] Houlin Zhao, Funke Opeke CEO, and MainOne. "Sharing networks, driving growth". In: Shar. networks, Driv. growth (2017). URL: https : / / www . itu . int/en/itunews / Documents / 2017 / 2017 06/2017\%7B\%5C_\%7DITUNews06-en.pdf.

[7] Ibrahim Alhassan Gedel and Nnamdi I. Nwulu. "Low Latency 5G Distributed Wireless Network Architecture :" in: (2021).

[8] Vanessa Vasconcellos and Paulo H Portela De Carvalho. "A Framework for Evaluating 5G Infrastructure Sharing with a Neutral Host". In: 28Th Conf. Fruct Assoc. (2020), pp. 660-663.

[9] Ovase Karim Kazi, Sajjad Ali Memon, Erum Saba, Zahid Ali, and Falak Naz. "Infrastructure Sharing and Remedies in Next Generation Cellular Networks". In: 20.12 (2020), pp. 184-192.

[10] Jose Marino Garcia and Tim Kelly. The Economics and Policy Implications of Infrastructure Sharing and Mutualisation in Africa. Tech. rep. 2015. URL: http : / / pubdocs . worldbank . org / pubdocs / publicdoc / 2016 / 1 / 533261452529900341 / WDR16 - BP - Infrastructure - Mutualisation Garcia.pdf.

[11] Andre Mendes Cavalcante, Maria Valeria Marquezini, Luciano Mendes, and Carlos Salle Moreno. "5G for Remote Areas: Challenges, Opportunities and Business Modeling for Brazil". In: IEEE Access 9 (2021), pp. 10829-10843. ISSN: 21693536. DOI: 10.1109/ACCESS . 2021. 3050742.

[12] Edvin Jonathan Kitindi, Catherine Francis Mangare, and Asif Kabir. "Infrastructure Sharing for Cellular Networks in Tanzania”. In: Int. J. Inf. Commun. Technol. Hum. Dev. 12.1 (2020), pp. 1-23. ISSN: 19355661. DOI: 10.4018/ijicthd. 2020010101.
[13] B. C. Lefevre. "Mobile Sharing”. In: 8th Glob. Symp. Regul. (2008).

[14] Tong Li and Lin Bai. "Model of wireless telecommunications network infrastructure sharing \& benefit-cost analysis". In: Proc. - 2011 4th Int. Conf. Inf. Manag. Innov. Manag. Ind. Eng. ICIII 20112 (2011), pp. 102-105. DoI: 10.1109/ICIII . 2011. 171.

[15] GSMA. "High Altitude Platform Systems". In: June (2021).

[16] Hong Ren, Nan Liu, Cunhua Pan, Maged Elkashlan, Arumugam Nallanathan, Xiaohu You, Lajos Hanzo, ) C Pan, M Elkashlan, and A Nallanathan. "Maximum Statistical Delay-Guarantee for Next-generation CRAN: An Effective Capacity Based Approach". In: Eecs.Qmul.Ac.Uk (2018), pp. 1-15. URL: http : / / www . eecs . qmul . ac . uk /\%7B \% 7Dnalla/paper / Ren $\% 7 B \% 5 C \_\% 7 D V T M \% 7 B \% 5 C \_\% 7 D 18$.pdf.

[17] Veronica Quintuna Rodriguez and Fabrice Guillemin. "Towards the deployment of a fully centralized Cloud-RAN architecture". In: 2017 13th Int. Wirel. Commun. Mob. Comput. Conf. IWCMC 2017 (2017), pp. 1055-1060. DOI: 10.1109/IWCMC . 2017.7986431.

[18] Ida Sèmévo TOGNISSE, Ahmed Dooguy KORA, and Jules DEGILA. "Cloud-RAN And Coverage Gap in Rural Areas". In: 10th 2021 IEEE Int. Conf. Commun. Network, Satell. (2021).

[19] Rajesh Gupta, Sudeep Tanwar, Sudhanshu Tyagi, and Neeraj Kumar. "Tactile internet and its applications in 5G era: A comprehensive review". In: Int. J. Commun. Syst. 32.14 (2019), pp. 1-49. ISSN: 10991131. DoI: 10.1002/dac. 3981.

[20] Ahmed Slalmi, Hasna Chaibi, Abdellah Chehri, Rachid Saadane, Gwanggil Jeon, and Nadir Hakem. "On the ultra-reliable and low-latency communications for tactile internet in 5G era". In: Procedia Comput. Sci. 176 (2020), pp. 3853-3862. ISSN: 18770509. DoI: $10.1016 / j$.procs. 2020.09 .003$. URL: https : / / doi . org / 10 . 1016/j . procs . 2020.09 .003$.

[21] Adrian Kliks, Bartosz Musznicki, Karol Kowalik, and Paweł Kryszkiewicz. "Perspectives for resource sharing in 5G networks". In: Telecommun. Syst. 68 (Aug. 2018). DoI: 10.1007/s11235-0170411-3.

[22] Meysam Masoudi, Shari Sofia Lisi, and Cicek Cavdar. "Cost-effective Migration towards Virtualized C-RAN with Scalable Fronthaul Design". In: IEEE Syst. J. (2020), pp. 1-12.

[23] Idriss Saleh Bachar, Ahmed Dooguy Kora, and Roger Marcelin Faye. "Radio access and Transmission models for universal service”. In: 6.3 (2017), pp. 1018-1024. 
[24] Navrati Saxena, Abhishek Roy, and Hanseok Kim. "Traffic-Aware Cloud RAN: A Key for Green 5G Networks". In: IEEE J. Sel. Areas Commun. 34.4 (2016), pp. 1010-1021. ISSN: 07338716. DOI: $10.1109 /$ JSAC . 2016. 2549438.

[25] Aleksandra Checko, Henrik L. Christiansen, Ying Yan, Lara Scolari, Georgios Kardaras, Michael S. Berger, and Lars Dittmann. "Cloud RAN for Mobile Networks - A Technology Overview". In: IEEE Commun. Surv. Tutorials 17.1 (2015), pp. 405-426. ISSN: 1553877X. DOI: 10 . 1109 / COMST . 2014 . 2355255.

[26] Rodrigo Izidoro Tinini, Daniel Macedo Batista, Gustavo Bittencourt Figueiredo, Massimo Tornatore, and Biswanath Mukherjee. "Low-latency and energy-efficient BBU placement and VPON formation in virtualized cloud-fog RAN". In: J. Opt. Commun. Netw. 11.4 (2019), B37-B38. ISSN: 19430639. DOI: $10.1364 / \mathrm{JOCN}$. 11.000B37.

[27] Marco Savi, Massimo Tornatore, and Giacomo Verticale. "Impact of Processing-Resource Sharing on the Placement of Chained Virtual Network Functions". In: c (2019). arXiv: arXiv:1710.08262v5.

[28] Liljana Gavrilovska, Valentin Rakovic, and Daniel Denkovski. "From Cloud RAN to Open RAN". In: Wirel. Pers. Commun. (2020).

[29] Aztelco. "Ifc/aspects techniques et de régulation/plan de développement." In: (2012).

[30] Capstone Team, S- Zachary Allin, Akash Patel, Mihail Stantchev, Luciano Taranto, and Jerry Wasdyke. "Cloud Radio Access Network ( C-RAN ): A Step Towards a 5G Wireless Network". In: 30.July (2015), p. 2015.

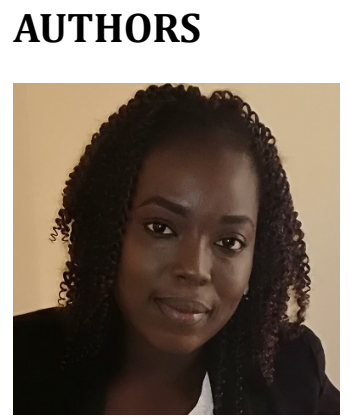

Ida Sèmévo TOGNISSE is a Ph.D. student at the Institute of Mathematics and Physics (IMSP), University of AbomeyCalavi (UAC), Benin. Specialised in digital radio broadcasting systems, she graduated in 2017 as a network design and telecommunication engineer from the Ecole Polytechnique d'Abomey-Calavi (EPAC). She earned a master's degree in information and communication technology from the Institute of Mathematics and Physical Science in 2018. For the past three years, she has been fully focused on research on universal access to mobile telephony and connectivity in rural and isolated areas.

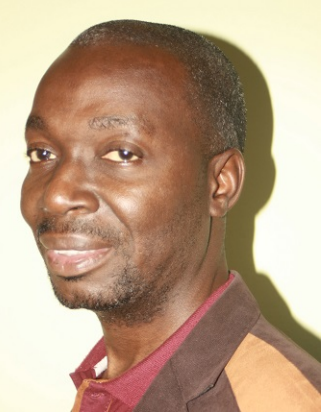

Ahmed D. KORA is IEEE senior member and ITU consultant. He received a Master "Réseaux Télécoms" degree from "Ecole Superieure Multinationale de Telecommunications" (ESMT) in 2003, and Ph.D degree in telecommunications from the University of Limoges, France, in 2007. He is a Professor with the ESMT where he is currently the head of teaching, training, and research. His research area covers communication and networks system architecture, free space optics, fiber optics, quality of service, universal access, artificial intelligence, SDN, etc.

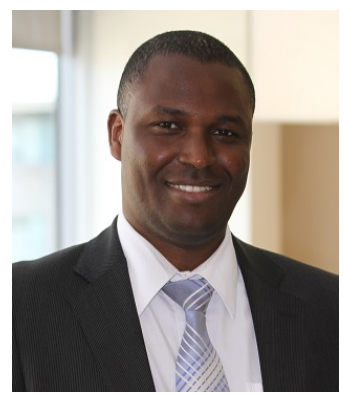

Jules DEGILA (Ph.D.) is a Senior Lecturer at the University of Abomey-Calavi, Benin. He is specialized in the optimization of telecommunication networks and systems architecture, deployment, and operations. During the last ten years, he has held different management, executive, and board member positions for Western and African telecommunication companies. A guest speaker at various universities in the fields of operations research and telecommunications, he has also advised many companies and governments as a strategist in real-time communications and Internet Protocol (IP) networking. From April 2005 to June 2010, he was an Assistant Director of Telecommunications Applications, and Technologies for a leading Canadian analog and digital television, high-speed Internet, and telephony services provider. During his career in Canada, he was responsible both for the technological architectures of all telecommunication services, and for exploring and developing advanced telecommunication applications and technologies. He served as a technology strategist for regulatory affairs, and was also a member of different working groups of CableLabs (a Denver, Colorado-based research consortium for cable operators). 